\title{
Hierarchical Construction of Finite Diabatic Sets, By Mathieu Functions
}

\author{
R. Englman ${ }^{a, b}$, A. Yahalom ${ }^{b}$ and M. Baer ${ }^{a}$ \\ ${ }^{a}$ Department of Physics an Applied Mathematics, \\ Soreq NRC, Yavne 81810,Israel \\ ${ }^{b}$ College of Judea and Samaria, Ariel 44284, Israel \\ e-mail: englman@vms.huji.ac.il; asya@ycariel.yosh.ac.il; \\ mmbaer@netvision.net.il;
}

November 20, 2018

\begin{abstract}
An extension is given for the standard two component model of adiabatic, Born-Oppenheimer (BO) electronic states in a polyatonic molecule, by use of Mathieu functions of arbitrary order. The curl or compatibility conditions for the construction of a diabatic set of states based on a finite- dimensional subset of BO states are not satisfied exactly. It is shown, however, that, by successively adding higher order Mathieu functions to the BO set, the compatibility conditions are satisfied with increasingly better accuracy. We then generalize to situations in which the nonadiabatic couplings (the dynamic corrections to the BO approximation) are small (though not necessarily zero) between a finite-dimensional $\mathrm{BO}$ subset and the rest of the $\mathrm{BO}$ states. We prove that approximate diabatic sets exist, with an error that is of the order of the square of the neglected nonadiabatic couplings.
\end{abstract}

PACS number(s):31.15.-p, 31.30.-i, 31.30.Gs, 31.70.-f

keywords: Born-Oppenheimer states, nonadiabatic coupling, Mathieu functions, diabatic set, Yang-Mills field 


\section{Background and Introduction, preceded by a Homage}

Some of the authors in the article have had the good fortune that their pathways crossed with those of Per-Olov Lowdin, be this in Slater's Group at MIT, in Menton or at the Sanibel Workshops. They keep the memory of an endowed and innovative researcher, as well as of a Scientist Statesman, who followed in the footsteps of another great Scandinavian, Niels Bohr.

The question of whether it is possible to effect in general an adiabatic to diabatic transformation (ADT) has been recently reopened [1],[2], [3]. One formulation of the issues [4]-[9] is whether a strictly diabatic basis in which linear combinations of a small number of Born-Oppenheimer electronic states are chosen in such a way that the nonadiabatic coupling between the transformed states vanishes exists.

In view of possible ambiguities let us define the terms:

$$
\zeta_{k} \equiv \zeta_{k}\left(\mathbf{x}_{\mathbf{e}}, X_{r}\right)
$$

is a solution of the Born-Oppenheimer wave-equation for the electronic part (electronic coordinates $\mathbf{x}_{\mathbf{e}}$, to be suppressed at a later stage), involving (as parameters) the nuclear coordinate set $\{X r\}$. For any given value of $\{X r\}$, the $\zeta_{k}$ form a complete set, known as the adiabatic set or Born-Oppenheimer electronic states, labeled by $k=1 . . \infty$. Let us consider only the finite set with $k=1, n$ (where $n$ is in practice $2-4$ ). We shall call this the $P$-set and designate its complement the $Q$ set. (This nomenclature follows previous practice to decompose the full Hilbert space into two disjoint subspaces, $P$ and $Q$ [10]. Then the projection operator $\mathbf{I}$ over the full Hilbert space can be written as the sum of two projection operators in the sub- spaces, namely $\mathbf{I}=\mathbf{P}+\mathbf{Q})$ The challenge has been to find a so-called diabatic set

$$
\xi_{m}=\xi_{m}\left(\mathbf{x}_{\mathbf{e}}, X_{r}\right)
$$

related to the $P$ adiabatic-set alone, such that the nuclear derivative term in the nuclear part of the Born-Oppenheimer equation corresponding to $\xi_{m}$ vanishes. Baer [11] showed that this can be achieved with an ADT matrix $A_{i j}\left(X_{r}\right)$ that satisfies

$$
A_{i j, r}+\tau_{i k}^{(r)} A_{k j}=0
$$

for $i, j, k$ in $P$ and all nuclear coordinate indexes $r$. (A tensor-algebra notation is used here, but supplementing the double-index summation convention 
with specification of the range of summands. The symbol $r$ after a comma represents, as usual in tensor algebra, differentiation with respect to $X_{r}$ ). Then

$$
\xi_{m}=\zeta_{k} A_{k m}
$$

$(k, m$ in $P)$ and the nuclear Schrödinger equation contains no first-order derivatives of the nuclear co-factors of $\xi_{k}$. In equation (3) the following integrals over the electronic coordinates appear:

$$
\begin{gathered}
\tau_{k m}^{(r)}=<\zeta_{k} \mid \zeta_{m, r}> \\
\tau_{k m}^{(r)}=-\tau_{m k}^{(r)}
\end{gathered}
$$

The initial value conditions for $A_{k m}$ for some chosen initial $\left\{X_{r}=X_{r 0}\right\}$ are

$$
A_{k m}\left(\left\{X_{r 0}\right\}\right)=\delta_{k m}
$$

This is a convenient set of initial conditions, which can, however, be generalized to cases when initially $A_{k m}$ is non-zero for any $k$ and $m$ both inside $P$, or both inside $Q$ [2]. Reference [11] also noted the following compatibility (curl) conditions as a requirement for the solution of equation (3) :

$$
\tau_{k m, s}^{(r)}-\tau_{k m, r}^{(s)}=\tau_{k n}^{(r)} \tau_{n m}^{(s)}-\tau_{k n}^{(s)} \tau_{n m}^{(r)}
$$

$(k, m, n$ in $P$; all unequal $r, s$. A shorthand way of writing this equation in terms of many-dimensional vector matrices is $\operatorname{curl} \tau=-\tau \mathbf{x} \tau$.) These relations arise from differentiation of equation (3) with respect to $X_{s}$, a further differentiation with respect to $X_{r}$ of another equation of the form equation (3) but involving $s$ instead of $r$, and subtraction. The actual requirement of compatibility is that

$$
\left(\tau_{k m, s}^{(r)}-\tau_{k m, r}^{(s)}\right) A_{m h}=\left(\tau_{k n}^{(r)} \tau_{n m}^{(s)}-\tau_{k n}^{(s)} \tau_{n m}^{(r)}\right) A_{m h}
$$

$(k, m, n, h$ in $P)$. Satisfaction of the relations in equation (8) is a sufficient condition for equation (9) to hold for all and any $A_{m h}$ and for the existence of solutions in equation (3) .

Having now formulated the general issue,we work out in the next section a case, involving Mathieu functions, in which equation (8) is not satisfied. These functions have already been used in the context of the ADT matrix [12]. We show here how the compatibility condition can be better and better satisfied by successively enlarging in a systematic way the finite subset (the 
$P$ subspace). Having illustrated with the Mathieu functions the case of small non-adiabatic coupling between $P$ and $Q$ subspaces, we then return in section 3 to the general theory and show what modifications are needed in the ADT matrix, generally, when the non-adiabatic coupling between $P$ and $Q$ are small, of order $\epsilon$. In section 5 we employ an analogy to show that, as in the case of the ADT matrix, frequently a large Hilbert-space is needed formally, but in practice a restricted space suffices.

\section{Mathieu Functions as Adiabatic States}

An electronic Schrödinger equation for the angular electronic coordinate $\theta$ and the polar nuclear coordinates $q$ and $\phi$ was written in Ref. [12], Eq. (1), as

$$
\left[-\frac{1}{2} E_{e l} \frac{\partial^{2}}{\partial \theta^{2}}-G(q) \cos (2 \theta-\phi)-u(q, \phi)\right] \Psi(\theta \mid q, \phi)=0
$$

where $E_{e l}$ is a characteristic electronic quantity, $G(q)$ is a nuclear-electronic interaction coefficient, frequently assumed to be proportional to the nuclear coordinate $q$ and $u(q, \phi)$ is the eigen-energy of the solution (being part of the adiabatic potential for the nuclear motion). Equation (10) is recognized as the Mathieu differential equation, which has enjoyed a wide literature and a variety of notations [14]-[16]. We shall use the solutions given by these sources (thus differing from the methodology in Ref. [12], in which the solutions were derived), but shall retain the symbols for the parameters introduced in Ref. [12]. However, we shall make life easier for the reader by giving the relations between the parameters. In the relations, we indicate the literature sources by adding as subscripts the initials of the authors surnames. In the spirit of Born-Oppenheimer approximation the small parameter in the theory is

$$
x \equiv \frac{G(q)}{E_{e l}}=\frac{k q}{E_{e l}}=8 q_{W W}=-q_{M L}=-\frac{h_{M F}^{2}}{4}
$$

and the adiabatic angular coordinate for the electronic motion relative to the nuclear one is

$$
z \equiv \theta-\frac{\phi}{2}
$$

The last relation implies that all derivatives with respect to $\phi$ can be

replaced by $-\frac{1}{2}$ times the derivative with respect to $z$. In the notation of 
Ref. [15], the two families of solutions of equation (10) that are of interest to us have the form:

$$
\begin{array}{ll}
c e_{2 n+1}(z,-x)=\sum_{r=0}^{\infty} A_{2 r+1}^{2 n+1}(-x) \cos (2 r+1) z & (n=0,1, . .) \\
s e_{2 n+1}(z,-x)=\sum_{r=0}^{\infty} B_{2 r+1}^{2 n+1}(-x) \sin (2 r+1) z & (n=0,1, . .)
\end{array}
$$

The functions are conventionally normalized to $\pi$, so that the squares of the coefficients sum up to 1 . Thus, when we use equations $(13-14)$ for the wave-functions $\Psi$ of equation (10) (that are normalized in the square to unity) each function has to be divided by $\sqrt{\pi}$. When we number the adiabatic electronic wave functions according to the order of their energy surfaces (as is commonly done in molecular physics) by $\left(m^{\prime}\right)$, we have the correspondences (for $x>0$ ):

$$
\begin{aligned}
& \Psi^{\left(1^{\prime}\right)}\left[\equiv \Psi^{(1)}(\theta \mid q, \phi)\right]=\frac{c e_{1}(z,-x)}{\sqrt{\pi}}, \Psi^{\left(2^{\prime}\right)}=\frac{s e_{1}(z,-x)}{\sqrt{\pi}}, \\
& \Psi^{\left(3^{\prime}\right)}=\frac{c e_{3}(z,-x)}{\sqrt{\pi}}, \Psi^{\left(4^{\prime}\right)}=\frac{s e_{3}(z,-x)}{\sqrt{\pi}}, \Psi^{\left(5^{\prime}\right)}=\frac{c e_{5}(z,-x)}{\sqrt{\pi}}, \ldots
\end{aligned}
$$

Solutions of equation (10) of the form $c e_{2 n}, s e_{2 n}$ have vanishing matrix elements with those in equation (15) and can be disregarded. For small $|x|$, the following are the leading terms in the expansions of the Mathieu functions for $n=0$ [15] :

$$
\begin{aligned}
& c e_{1}(z,-x)=\left(1-\frac{x^{2}}{128}\right) \cos (z)+\frac{x}{8}\left(1-\frac{x}{8}\right) \cos (3 z)+\frac{x^{2}}{192} \cos (5 z)+\ldots \\
& \operatorname{se}_{1}(z,-x)=\left(1-\frac{x^{2}}{128}\right) \sin (z)+\frac{x}{8}\left(1+\frac{x}{8}\right) \sin (3 z)+\frac{x^{2}}{192} \sin (5 z)+\ldots
\end{aligned}
$$

Still for small $|x|$, the following are the leading terms in $A_{2 r+1}^{2 n+1}(-x)$ and $B_{2 r+1}^{2 n+1}(-x)[14,15,17]$ : for $r \geq n$ :

$$
\frac{(2 n+1) !}{[(r-n) !(r+n+1) !]}\left(\frac{x}{4}\right)^{r-n}+O\left(x^{r-n}\right)
$$

for $r<n$

$$
\frac{(n-r) !}{[(n-r) !(2 n) !]}\left(-\frac{x}{4}\right)^{n-r}+O\left(x^{n-r}\right)
$$


Now the eigenvalues $u$ of equation (10) are of the order of $\frac{(2 n+1)^{2} E_{e l}}{2}$ $[12,15,16]$ (this can be seen from the leading terms of equation (13) and equation (14) in which equation (18) and equation (19) were taken into consideration), therefore the $n=0$ pair is well separated energetically from the $n>0$ states that lie higher. Adopting the nomenclature of section 1, we shall call the two states in equation (13) and equation (14) with $n=0$ the $P$ subset. For $x=0, P$ is doubly degenerate; for small $|x|$ it is nearly degenerate. We look at the nonadiabatic coupling matrices $\tau$ within this subset. Using the definitions shown in equation (5) for $r=q$ and $\phi$ (see also equation (15) in Ref. [12]) and the expansion in equation (16) and equation (17) we obtain:

$$
\tau_{1^{\prime} 2^{\prime}}^{(q)}=0
$$

and

$$
\tau_{1^{\prime} 2^{\prime}}^{(\phi)}=-\frac{\left(1+\frac{x^{2}}{32}\right)}{2 q}
$$

agreeing with equation(16) in Ref.[12]. Turning now to our equation (8) , we write the curl-term on the left hand side in the present curvilinear, plane-polar coordinate system as:

$$
\frac{1}{q}\left[\frac{\partial\left(q \tau^{(\phi)}\right)}{\partial q}-\frac{\partial\left(\tau^{(q)}\right)}{\partial \phi}\right]=-\frac{x^{2}}{32 q^{2}}=-\frac{k^{2}}{32 E_{e l}^{2}}
$$

where we have used equation (11) and equations $(21,20)$ in the last result.

However, evaluation of the right hand side of equation (8),$-\tau \times \tau$, inside the $P$-set $\left(1^{\prime}, 2^{\prime}\right)$ shows that this is zero. Clearly, the missing part comes from the $P-Q$ inter-set nonadiabatic coupling terms $\tau_{J \alpha}^{(r)}$. When we evaluate $-\tau \times \tau$ to the lowest order in $x$, we find that this exactly matches the curl-value and that the missing value come from the $n=1$ functions $c e_{3}$ and $s_{3}$. This means that enlarging the $P$ set from $n=0$ to $n=0,1$ ensures the compatibility conditions for the solution of the ADT matrix, correct to the order of $E_{e l}^{-2}$. To satisfy the curl-conditions to higher powers in $E_{e l}^{-1}$ (or $x$ ) will require bringing in higher $n$ values. It will be presently shown that each higher power of $x^{2}$ requires one further $n$.

Table 1 shows the leading values for small $|x|$, as obtained from equation (18) and equation (19) .

We now turn to the nonadiabatic couplings $\tau^{(r)}(J, \alpha)=-\tau^{(r)}(\alpha, J)$, where $J$ is one of the $n=0$ and $\alpha$ is one of $n>0$. Since we calculate these correct only to $x$, we find from table 1 , upon recalling the power series expansions equation (16) and equation (17) for $c e_{1}$ and $s e_{1}$ and the orthogonality 


\begin{tabular}{|c|c|c|c|c|}
\hline \hline$r / n$ & 0 & 1 & 2 & 3 \\
\hline \hline 0 & 1 & $\frac{x}{8}$ & $O\left(x^{2}\right)$ & $O\left(x^{3}\right)$ \\
\hline 1 & $-\frac{x}{8}$ & 1 & $\frac{x}{16}$ & $O\left(x^{2}\right)$ \\
\hline
\end{tabular}

Table 1: Some leading terms in the coefficients $A$ and $B$ for small $|x|$

of the trigonometric functions, that only $n=1$ contributes nonvanishingly. We now draw up the list of the nonadiabatic couplings labeled, express them in terms of quantum mechanical bra-kets of the derivatives and give their values. We give those values of $r$ in the expansions equation (13) and equation (14), which contribute.

$$
\begin{aligned}
& \tau_{1^{\prime} 3^{\prime}}^{(q)}=\quad<c e_{1}\left|\frac{\partial}{\partial q}\right| c e_{3}>\approx-\frac{x}{8 q} \quad(r=0) \\
& \tau_{1^{\prime} 4^{\prime}}^{(\phi)}=-\frac{1}{2}<c e_{1}\left|\frac{1}{q} \frac{\partial}{\partial z}\right| s e_{3}>\approx-\frac{x}{8 q} \quad(r=0,1) \\
& \tau_{2^{\prime} 4^{\prime}}^{(q)}=\quad<s e_{1}\left|\frac{\partial}{\partial q}\right| s e_{3}>\approx-\frac{x}{8 q} \quad(r=1) \\
& \tau_{2^{\prime} 3^{\prime}}^{(\phi)}=-\frac{1}{2}<c e_{2 n+1}\left|\frac{1}{q} \frac{\partial}{\partial z}\right| s e_{1}>\approx \frac{x}{8 q} \quad(r=0,1)
\end{aligned}
$$

From these we calculate the vectorial cross-product in the ground $[P=$ $\left.\left(1^{\prime}, 2^{\prime}\right)\right]$ doublet

$$
(\tau \times \tau)_{1^{\prime} 2^{\prime}}=\sum_{\alpha \in Q}\left[\tau^{(q)}\left(c e_{1}, \alpha\right) \tau^{(\phi)}\left(\alpha, s e_{1}\right)-\tau^{(\phi)}\left(c e_{1}, \alpha\right) \tau^{(q)}\left(\alpha, s e_{1}\right)\right]
$$

Collecting all matrix elements, after noting the antisymmetric character of the coupling matrices and the negative sign before second term in equation (24), one finds finally

$$
(\tau \times \tau)_{1^{\prime} 2^{\prime}}=\frac{x^{2}}{32 q^{2}}
$$

This agrees with the negative of the curl, as required. The contributing intermediate states are seen to be the lowest energy set from $Q(n=1)$, which is energetically immediately above the $P(n=0)$ set. This balances the $x^{2}$ term in the curl, equation (22). More generally, as one goes in $c e_{1}$, $s e_{1}$ (or in $\Phi^{\left(1^{\prime}\right)}, \Phi^{\left(2^{\prime}\right)}$ ) to higher approximations in $x$, curl $\tau_{1^{\prime} 2^{\prime}}$ will contain higher powers of $x^{2}$. (Recall that $x \propto \frac{1}{E_{e l}}$ is the small parameter in the BornOppenheimer approximation.) We wish to show now that each consecutive term of order $x^{2 s}$ in the expansion of $\operatorname{curl} \tau_{1^{\prime} 2^{\prime}}$ will be balanced by a further set of the higher energy wave functions, precisely up to $c e_{2 s+1}, s e_{2 s+1}$ and none higher. (Since the method of computing the matrices is laborious, we 
do not calculate the higher order terms in the curl or in the vector product $(\tau \times \tau)_{1^{\prime} 2^{\prime}}$, only show from where the latter arise.) The proof relies on the substitution of the dominant term, given in equation (18) and equation (19) , into the expansions (13)-(14) for the Mathieu functions. We thus have approximately, for small $|x|$,

$$
\begin{array}{ll}
c e_{2 n+1}(z,-x) \approx \sum_{r=0}^{\infty} a_{2 r+1}^{2 n+1} x^{|n-r|} \cos (2 r+1) z & (n=0,1, . .) \\
\operatorname{se}_{2 n+1}(z,-x) \approx \sum_{r=0}^{\infty} b_{2 r+1}^{2 n+1} x^{|n-r|} \sin (2 r+1) z & (n=0,1, . .)
\end{array}
$$

where $a$ and $b$ are (in the leading order) numerical. Next we form the nonadiabatic coupling vector-matrix $\tau$, whose elements are proportional to:

$$
\begin{aligned}
<c e_{1}\left|\frac{\partial}{\partial q}\right| c e_{2 n+1}>\approx \frac{1}{q} \sum_{r=0}^{\infty} a_{2 r+1}^{1} a_{2 r+1}^{2 n+1}|n-r| x^{|n-r|+r} \\
<c e_{1}\left|\frac{1}{q} \frac{\partial}{\partial z}\right| s e_{2 n+1}>\approx \frac{1}{q} \sum_{r=0}^{\infty} a_{2 r+1}^{1} b_{2 r+1}^{2 n+1}(2 r+1) x^{|n-r|+r}
\end{aligned}
$$

and two further matrix elements of similar form for coupling with $s e_{1}$. To compensate the term of order $x^{2 s}$ in $\operatorname{curl} \tau_{1^{\prime} 2^{\prime}}$ by a similar term in $(\tau \times \tau)_{1^{\prime} 2^{\prime}}$, we require that

$$
|n-r|+r=s
$$

Two cases have to be considered: $n \geq r$ and $n<r$. In the former, equation (30) gives $n=s$. In the latter case, one gets from equation (30) after transposing

$$
2 r=n+s
$$

If $n$ were greater than $s$, then

$$
2 r=n+s<2 n
$$

contradicting the assumption that $n<r$. Thus, in all cases, only Mathieu functions of up to order $n=s$ need to be added to the intermediate set to compensate the $x^{2 s}$ term in the curl; higher order functions start with higher powers of $x$. This leads to a hierarchical extension of the $P$ set to ensure the compatibility condition to successively better accuracy in the ground, $\left(1^{\prime}, 2^{\prime}\right)$ set. 


\section{The Projected ADT Matrix}

We return now to the general case embodied in equation (8) and equation (9) .

It has been established (Ref. [11], Appendix 1) that if in equation (8) the summation over $n$ is extended to $P+Q$, i.e. the intermediate states run over the complete set, then equation (8) is satisfied for $\tau_{k m}^{(r)}$ defined by (5)-(6).

We shall now consider cases in which all $\tau_{k m}^{(r)}$ are small, (say) of order $\epsilon$, for either $k$ or $m$ being in the set $Q$ complementary to $P$, though they can be large for both $k$ and $m$ being in $P$ or in $Q$. Physical conditions for the existence of such situations have been noted before as either large energy gaps between $P$ and $Q$ ([5], section III.A, end), or a strong vibronic coupling localized in the neighborhood of some reference configuration ([5], section III.B), with which the $Q$-set has small overlap. In section 4 we consider yet another situation, where the smallness of $\epsilon$ arises from the small ratio between electronic and nuclear masses.

Our aim is to show that equation (3) and equation (4) continue to be approximately valid, even though equation (8) is not formally satisfied. Our demonstration should disarm objections to some practical uses of the ADT matrix. It also extends a previous proof [13], that the diabatic set exists when the above considered $\tau_{k m}^{(r)}$ are exactly zero, to those more frequently encountered situations, where the non adiabatic couplings are non-zero, but small.

Notation: Henceforth we shall use Latin subscripts (e.g., $j, k, m, .$.$) for$ the total Hilbert space, capital Latin subscripts (e.g., $J, K, M, .$. ) for the $P$ subset, and Greek subscripts (e.g., $\alpha, \beta, .$.$) for the Q$ subset. In this notation $\tau_{J \alpha}^{(r)}$ are all of order $\epsilon$ and small. We now write out equations (3) with initial conditions (7) for the full Hilbert space. Solutions of these exist, since the curl-condition in (8) is satisfied for the full Hilbert-space. Explicitly, (3) reads as follows:

$$
A_{J K, r}+\tau_{J M}^{(r)} A_{M K}+\tau_{J \alpha}^{(r)} A_{\alpha K}=0
$$

for derivatives fully within $P$, and

$$
A_{\alpha K, r}+\tau_{\alpha M}^{(r)} A_{M K}+\tau_{\alpha \beta}^{(r)} A_{\beta K}=0
$$

for the inter - $P Q$ matrix element derivatives, and a further equation (which will not be of interest to us) for the intra- $Q$ derivative matrix elements. 
Noting that at $\left\{X_{r}\right\}=\left\{X_{r 0}\right\}$ the initial conditions (7) hold, we assume now that the matrix elements at a general point $\left\{X_{r}\right\}$ close to it can be expanded in powers of $\left\{X_{r}-X_{r 0}\right\}$ as a Taylor series. The condition for this assumption is that the matrix elements $\tau_{k m}^{(r)}$ have no singularities in the neighborhood considered [2]. The expansion takes the following form:

$$
\begin{aligned}
& A_{j k}\left(\left\{X_{r}\right\}\right)=\sum_{N=0}^{\infty} \frac{1}{N !}\left[\sum_{r_{1}=1}^{M} \ldots \sum_{r_{N}=1}^{M}\left(X_{r_{1}}-X_{r_{1} 0}\right) \ldots\left(X_{r_{N}}-X_{r_{N} 0}\right)\right. \\
& \left.A_{j k, r_{1} \ldots r_{N}}\left(\left\{X_{r 0}\right\}\right)\right]
\end{aligned}
$$

in which the coefficients contain the derivatives $A_{j k, r_{1} \ldots r_{N}}\left(\left\{X_{r 0}\right\}\right)$ to the $N^{\prime}$ th order $\left(r_{1} \ldots r_{N}\right.$ contains $N$ similar or dissimilar symbols referring to the nuclear coordinates, and $M$ is the number of nuclear coordinates). We shall find that all inter- $P Q A_{\alpha J}$ are of order $\epsilon$, but the corrections to the intra- $P$ $A_{J K}$ are merely of order $\epsilon^{2}$.

To start, we prove this for the first derivatives $A_{J K, r}\left(\left\{X_{r 0}\right\}\right)$ and $A_{\alpha K, r}\left(\left\{X_{r 0}\right\}\right)$ respectively. These can be immediately evaluated from equation (33) and equation (34), with the following results: In equation (33), at $\{X=X r 0\}$ the third term is zero by the initial conditions $(7) A_{k m}\left(\left\{X_{t 0}\right\}\right)=$ $\delta_{k m}$, and this gives the second term only, which has the same result as ignoring the $Q$ set, that is:

$$
\begin{aligned}
& A_{J K, r}\left(\left\{X_{t 0}\right\}\right)+\tau_{J M}^{(r)}\left(\left\{X_{t 0}\right\}\right) A_{M K}\left(\left\{X_{t 0}\right\}\right)= \\
& A_{J K, r}\left(\left\{X_{t 0}\right\}\right)+\tau_{J K}^{(r)}\left(\left\{X_{t 0}\right\}\right)=0 .
\end{aligned}
$$

In equation (34) the third term is similarly zero by the initial conditions, thus:

$$
\begin{aligned}
& A_{\alpha K, r}\left(\left\{X_{t 0}\right\}\right)+\tau_{\alpha M}^{(r)}\left(\left\{X_{t 0}\right\}\right) A_{M K}\left(\left\{X_{t 0}\right\}\right)= \\
& A_{\alpha K, r}\left(\left\{X_{t 0}\right\}\right)+\tau_{\alpha K}^{(r)}\left(\left\{X_{t 0}\right\}\right)=0
\end{aligned}
$$

we conclude that $A_{\alpha K, r}\left(\left\{X_{t 0}\right\}\right)=-\tau_{\alpha K}^{(r)}\left(\left\{X_{t 0}\right\}\right)$ is of the same order of $\epsilon$, due to $\tau_{\alpha K}^{(r)}\left(\left\{X_{t 0}\right\}\right)$ being of this order.

In the appendix we establish by mathematical induction that the corrections to all the inter- $P Q$ set derivatives $A_{\alpha K, r_{1} \ldots r_{N}}\left(\left\{X_{t 0}\right\}\right)$ are of order $\epsilon$, and that the corrections to the intra-P derivatives $A_{J K, r_{1} \ldots r_{N}}\left(\left\{X_{t 0}\right\}\right)$ are of order $\epsilon^{2}$. Subsequent derivatives are obtained recursively, rather than through integration, so that the validity of the procedure depends on the 
convergence of the Taylor series (which is assumed to hold close to the initial point).

Summarizing, subject to the assumption of small (though not necessarily zero) inter- $P Q$ non-adiabatic coupling and analyticity of the matrix elements, the original procedure of Ref. [11] restricted to a finite dimensional set $(P)$ is approximately valid, and correct to order $\epsilon^{2}$, no matter that the compatibility conditions are not exactly satisfied. Earlier, it was shown, for the model discussed here, that the discrepancy in the compatibility conditions (8) is $\epsilon^{2}$ (e.g., Ref. $[5,9]$ ); our result establishes the magnitude of error in the solution.

\section{An Inductive Proof}

We now establish by mathematical induction that the corrections to all the inter- $P Q$ set derivatives $A_{\alpha K, r_{1} \ldots r_{N}}\left(\left\{X_{t 0}\right\}\right)$ are of order $\epsilon$, and that the corrections to the intra- $P$ derivatives $A_{J K, r_{1} \ldots r_{N}}\left(\left\{X_{t 0}\right\}\right)$ are of order $\epsilon^{2}$.

This result was already proven for $N=1$. We assume that the result is correct for $N-1$ and show that the result is also correct for $N$. We can write:

$$
A_{\alpha K, r_{1} \ldots r_{N}}\left(\left\{X_{t 0}\right\}\right)=\partial_{r_{1} \ldots r_{m-1} r_{m+1} \ldots r_{N}} A_{\alpha K, r_{m}}\left(\left\{X_{t 0}\right\}\right)
$$

Using equation (34) this can be written also as:

$$
A_{\alpha K, r_{1} \ldots r_{N}}\left(\left\{X_{t 0}\right\}\right)=-\partial_{r_{1} \ldots r_{m-1} r_{m+1} \ldots r_{N}}\left(\tau_{\alpha M}^{\left(r_{m}\right)} A_{M K}+\tau_{\alpha \beta}^{\left(r_{m}\right)} A_{\beta K}\right)\left(\left\{X_{t 0}\right\}\right)
$$

Or also as:

$$
\begin{aligned}
A_{\alpha K, r_{1} \ldots r_{N}}\left(\left\{X_{t 0}\right\}\right) & =-\partial_{r_{1} \ldots r_{m-1} r_{m+1} \ldots r_{N}}\left(\tau_{\alpha M}^{\left(r_{m}\right)} A_{M K}\right)\left(\left\{X_{t 0}\right\}\right) \\
& -\partial_{r_{1} \ldots r_{m-1} r_{m+1} \ldots r_{N}}\left(\tau_{\alpha \beta}^{\left(r_{m}\right)} A_{\beta K}\right)\left(\left\{X_{t 0}\right\}\right)
\end{aligned}
$$

The first term in the right hand side of the above equation is proportional to $\tau_{\alpha M}^{\left(r_{m}\right)}$ and its derivatives, and thus by assumption is of order $\epsilon$. The second term in the right hand side of the above equation contains derivatives up to order $N-1$ of $A_{\beta K}$ and thus by the assumption of the induction is also of order $\epsilon$. Thus we established that the right hand side of equation (40) is of order $\epsilon$ and also $A_{\alpha K, r_{1} \ldots r_{N}}\left(\left\{X_{t 0}\right\}\right)$ is of order $\epsilon$. We conclude that $A_{\alpha K, r_{1} \ldots r_{N}}\left(\left\{X_{t 0}\right\}\right)$ is of order $\epsilon$ for all $N$ by induction.

Next we want to establish that correction to $A_{J K, r_{1} \ldots r_{N}}\left(\left\{X_{t 0}\right\}\right)$ are of order $\epsilon^{2}$. We established this result for $N=1$ inside the text. We proceed 
by assuming that this result is true for $N-1$ and will prove its correctness for $N$, again we write:

$$
A_{J K, r_{1} \ldots r_{N}}\left(\left\{X_{t 0}\right\}\right)=\partial_{r_{1} \ldots r_{m-1} r_{m+1} \ldots r_{N}} A_{J K, r_{m}}\left(\left\{X_{t 0}\right\}\right)
$$

Using equation (33) we obtain:

$$
A_{J K, r_{1} \ldots r_{N}}\left(\left\{X_{t 0}\right\}\right)=-\partial_{r_{1} \ldots r_{m-1} r_{m+1} \ldots r_{N}}\left(\tau_{J M}^{\left(r_{m}\right)} A_{M K}+\tau_{J \beta}^{\left(r_{m}\right)} A_{\beta K}\right)\left(\left\{X_{t 0}\right\}\right)
$$

Or also as:

$$
\begin{aligned}
A_{J K, r_{1} \ldots r_{N}}\left(\left\{X_{t 0}\right\}\right) & =-\partial_{r_{1} \ldots r_{m-1} r_{m+1} \ldots r_{N}}\left(\tau_{J M}^{\left(r_{m}\right)} A_{M K}\right)\left(\left\{X_{t 0}\right\}\right) \\
& -\partial_{r_{1} \ldots r_{m-1} r_{m+1} \ldots r_{N}}\left(\tau_{J \beta}^{\left(r_{m}\right)} A_{\beta K}\right)\left(\left\{X_{t 0}\right\}\right)
\end{aligned}
$$

The first term in the right hand side of the above equation contains intra-P terms which are not considered corrections. The second term in the right hand side of the above equation, which is considered a correction term, contains derivatives up to order $N-1$ of $A_{\beta K}$, which are of order $\epsilon$ according to the theorem established above. When multiplied by derivatives of $\tau_{\alpha M}^{\left(r_{m}\right)}$ which are of order $\epsilon$ by our assumptions, the correction terms obtained is thus of order $\epsilon^{2}$ for $\mathrm{N}$. Thus the result holds for any $N$, which is what we have set out to prove.

\section{An Analogy}

Very frequently one has a complicated Hamiltonian $H$, which has non- zero matrix elements $H_{n m}$ between the components (designated $n$ and $m$ ) in an extended Hilbert space. For many practical purposes, e.g. for numerical work, one uses a restricted, finite set (belonging to the $P$ subset of the Hilbert space), such that $P-Q$ matrix elements of the Hamiltonian are small. The procedure of solving the Schrödinger equation in this manner can be formally written as:

$$
H \mathbf{P}=E_{P} \mathbf{P}
$$

where, as before, $\mathbf{P}$ is a projection operator and $E_{P}$ represents the set of eigenvalues corresponding to the set of solutions thus obtained. Premultiplication with $\mathbf{Q}$, gives

$$
\mathbf{Q} H \mathbf{P}=E_{P} \mathbf{Q P}=0
$$


since $P$ and $Q$ are disjoint. This implies that the procedure requires, for consistency, that $\mathbf{Q} H \mathbf{P}=0$, or that there be no matrix elements $H_{n m}$ of the Hamiltonian connecting the $Q$ and $P$ sets. While this is, in general, a true requirement for exact solutions, in practice, when one seeks meaningful approximate results, one is content with the smallness of these matrix elements. We have here a further example of a rigorous condition being inconsistent with an approximate method.

\section{Conclusion}

In section 2 we have seen that for adiabatic electronic wave functions in the form of Mathieu functions the compatibility (or curl) conditions are not satisfied but, by successively enlarging the finite set, the conditions can be satisfied to increasingly better accuracy. As previously shown in (e.g.) [5] Eq. (2.54), a non-zero curl gives rise to a "gauge field tensor, the nonAbelian analog of the electromagnetic field", also called the Yang-Mills field [18]. The use of Mathieu functions will thus enable a systematic study of the properties of this field in a molecular physical context [19].

We then discussed the question of the existence of a finite sized ADT matrix $A$ (which is distinct from the question of its uniqueness or single valuedness), being the solution of a finite number of partial differential equations that fail to satisfy compatibility conditions. We have started with a complete set, for which the existence of solution is assured. Under conditions that the nonadiabatic coupling with the exterior of the set is small, we have truncated the size of the set and removed part of the finite sized ADT matrix $A$. The compatibility conditions are not met within the finite set, but the remainder of $A$ satisfies the original differential equation approximately, namely to an accuracy which goes as the square of the neglected nonadiabatic coupling. We conclude that the failure of the compatibility conditions yet permits the existence of an approximate ADT matrix (subject to smallness of some nonadiabatic coupling terms).

\section{References}

[1] M. Baer and A. Alijah, Chem. Phys. Letters, 319, 489 (2000)

[2] M. Baer, Chem. Phys. 259123 (2000)(Especially section 3.2.2) 
[3] M. Baer, S.H. Lin, A. Alijah, S. Adhikari and G.D. Billing, Phys. Rev. A 62 03256-1 (2000); S. Adhikari, G.T. Billing, A. Alijah, S.H. Lin and M. Baer, Phys. Rev. A 62 03257-1 (2000)

[4] V. Sidis in M. Baer and C.Y. Ng (Editors), State Selected and Stateto-State Ion-Molecule Reaction Dynamics: Part, Theory (Wiley, New York, 1992); Adv. Chem. Phys. 8273 (2000)

[5] T. Pacher, L.S. Cederbaum and H. Koppel, Adv. Chem. Phys. 84, 293 (1993)

[6] D.R. Yarkony, Rev. Mod. Phys. 68, 985 (1996); Adv. At. Mol. Phys. 31, 511 (1998)

[7] Y.S. Wu and A. Kuppermann, Chem. Phys. Letters 235, 105 (1996)

[8] L.S. Cederbaum, J. Schirmer and H.D. Meyer, J. Phys. A 22, 2427 (1989)

[9] A. Thiel and H. Koppel, J. Chem. Phys. 110, 9371 (1999)

[10] H. Feshbach, Ann. Phys. (N.Y.) 5, 357 (1958)

[11] M. Baer, Chem. Phys. Letters, 35, 112 (1975)

[12] M. Baer, A. Yahalom and R. Englman, J. Chem. Phys. 109 , 6550 (1998)

[13] M. Baer, Chem. Phys. Letters, 322, 520 (2000)

[14] E. T. Whittaker and G.N. Watson, A Course in Modern Analysis (University Press, Cambridge 1927)

[15] N.W. MacLachlan, Theory and Application of Mathieu Functions (Clarendon Press, Oxford, 1947)

[16] P. M. Morse and H. Feshbach, Methods of Theoretical Physics (McGraw-Hill, New York, 1953) Vol. II, Section 11.2

[17] N. Davis, Phil. Mag. 31, 283 (1941)

[18] C.N. Yang and R. Mills, Phys. Rev. 96191 (1954)

[19] J. Moody, A. Shapere and F. Wilczek, Phys. Rev. Lett. 56893 (1986) 